\title{
Safety assurance through advances in long-term operation
}

\author{
Kevin James Mottershead ${ }^{1, *}$, Christian Robertson ${ }^{2}$, Sebastian Lindqvist ${ }^{3}$, \\ Francisco Javier Perosanz Lopez ${ }^{4}$, and Eija Karita Puska ${ }^{3}$ \\ ${ }^{1}$ Materials Science \& Structural Integrity, Wood, Warrington, UK \\ 2 DEN-DMN, CEA, Saclay, France \\ ${ }^{3}$ VTT, Espoo, Finland \\ 4 Structural Materials Division, Technology Department, Ciemat, Madrid, Spain
}

Received: 5 April 2019 / Accepted: 4 June 2019

\begin{abstract}
Mindful of the challenges to long-term operation, especially the severe safety and environmental consequences shown through historical nuclear power plant accidents (e.g. Fukoshima, Chernobyl, etc), it is imperative that European research and innovation focuses on demonstrating reliable long-term operation. Five examples of European Commission supported projects meeting such objectives are INCEFA+, SOTERIA, ATLAS + , MEACTOS and NUGENIA + . There are economies of scale within, and synergies across these projects which enable further advantage to be gained. Additionally, since researchers are well engaged internationally, this brings into European Organisations latest developments in understanding from further afield (e.g. USA, Japan), further enabling safety assurance advances, and enabling work overseas to be influenced consistent with European requirements. Through examples, this paper provides evidence of the advances claimed, whilst being careful to also declare areas of interest for which further work is still a priority.
\end{abstract}

\section{Introduction}

This paper presents evidence of the advances gained from selected European Commission supported Horizon2020 and FP7 projects, supporting long-term operation of nuclear power plant. The paper begins by briefly introducing the projects. Nuclear industry operational issues leading to long-term operation challenges are then described. These challenges are summarised next, together with examples of how the EC supported project portfolio has combined to meet some of these. The paper concludes with a summary of the challenges remaining, and activities underway to meet them.

\section{The EC supported project portfolio}

The authors of this paper are coordinators of five EC supported projects, four current, and one complete. These are described briefly here, and their relevance to long-term operation challenges is summarised later.
- INCEFA $+{ }^{1}$ (INcreasing safety in nuclear power plants by Covering gaps in Environmental Fatigue Assessment) began work in July 2015 (though the consortium had been together on an in-kind basis since 2013). 16 organisations participate in this project, which is funded at $€ 2.5 \mathrm{M}$ over 5 years from the EC, and in excess of $€ 3.6 \mathrm{M}$ from national sponsors. This project's focus is on creation of new environmental fatigue data aimed at improving understanding of fatigue sensitivity to three common parameters of interest, namely, effects of surface finish, hold time and mean stress. The objective is the creation of assessment rules that are able to predict fatigue lives which are more consistent with plant experience than is the case for present ASME/USNRC guidance. The project will reduce assessment conservatism through the creation of more reliable consistent data than has hitherto been available; this is through partners working to an agreed test protocol, and using common material specimens all made in the same facility. Detailed material and specimen characterisation data are collected to help understand data outliers.

\footnotetext{
* e-mail: kevin.mottershead@woodplc.com
} 
- SOTERIA ${ }^{2}$ (Safe lOng TERm operation of light water reactors based on Improved understanding of rAdiation effects in nuclear structural materials) began work in September 2015, building on many years of collaboration for consortium members within previous projects. 23 organisations work in this project, which is funded at $€ 5 \mathrm{M}$ over 4 years from the EC, and in excess of $€ 1 \mathrm{M}$ from national sponsors. The project is developing understanding of ageing phenomena in reactor pressure vessel steels and reactor internals. Experiments are performed to explore flux and fluence effects, effects of metallurgical heterogeneities, and environmental effects on materials ageing behaviours. Modelling tools are developed to help with assessment of structural components, based on the developed understanding.

- MEACTOS ${ }^{3}$ (Mitigating Environmentally Assisted Cracking (EAC) Through Optimization of Surface condition) started in September 2017 and runs for 4 years. 16 organisations participate and the EC supports the project with $€ 2.5 \mathrm{M}$ funding, with greater than $€ 1.5 \mathrm{M}$ national sponsor funding. This project will quantify the effect of various surface treatment techniques on the EAC behaviour of nuclear primary circuit structural materials, with the objective of developing practical guidelines suitable for incorporation in nuclear design and manufacturing codes. SCC testing is done using specimens with a variety of surface finishes. Significant demonstration of machining procedures, applied successfully in industries such as aeronautics or automotive to mitigate against SCC, is included in the test programme. - ATLAS $+{ }^{4}$ (Advanced Structural Integrity Assessment Tools for Safe Long Term Operation) began in June 2017 and runs for 4 years. 19 organizations collaborate with $€ 4 \mathrm{M}$ EC funding, and more than $€ 3.2 \mathrm{M}$ from national sponsors. Five different innovative large scale experiments are planned to generate data for validation of advanced modelling tools for application to nuclear piping systems and associated components. Modelling tool development is focussed on simulation and assessment of weld residual stresses and prediction of large ductile tearing. Assessment of safety margins using probabilistic methods is also being explored.

- NUGENIA $+{ }^{5}$ ran from September 2013 to September 2016. The project comprised two parts. Part 1 was concerned with optimising the way NUGENIA is managed such that it could fill the role of the European Commission's chosen integrator of Research and Development focussed on safety of existing Gen II and future

\footnotetext{
2 This project has received funding from the Euratom Research \& Training programme 2014-2018 under grant agreement $\mathrm{N}^{\circ} 661913$. The project website is http://soteria-project.eu

3 This project has received funding from the Euratom H2020 programme 2014-2018 under grant agreement No 755439. The project website is https://meactos.eu

${ }^{4}$ This project has received funding from the Euratom H2020 programme under grant agreement No 754589. The public website is under construction.

${ }^{5}$ This project has received funding from the Euratom Research \& Training programme 2007-2013 under grant agreement No604965. The NUGENIA website is http://nugenia.org
}

Gen III nuclear installations. During Part 2, there was a call for proposals for small pilot projects, and 13 projects were chosen (with $50 \% \mathrm{EC}$ funding totalling $€ 2.6 \mathrm{M}$ ) and managed under NUGENIA + . The chosen pilot projects addressed subject areas encompassing materials analysis, fluid dynamics modelling, materials forming, inspection, materials degradation, soil mechanics, test optimisation, and test data management.

\section{The nuclear industry operational issues}

The issues leading to long-term operational challenges can be categorised as economic, engineering, legislative, and safety.

\subsection{Economic issues}

Reference [1] provides a good general summary of the upto-date position for electricity generation in Europe, and the role of nuclear power in this. Presently, the nuclear capacity being retired, through either life expiry or political pressure, significantly exceeds the capacity under construction. As a result, forecasts are for European nuclear generation capacity to reduce, at least in the period to 2030. The effects of this reducing capacity, on confidence in electrical generation capacity, are further compounded by (a) retirements of fossil fuelled capacity driven by environmental concerns, (b) uncertainties in security of supply for the significant remaining fossil fuels imported from outside Europe, and (c) significant delays bringing new nuclear generation capacity into service throughout Europe. Thus, there are clearly strong economic drivers to keep as much as possible of the existing European Nuclear capacity running for as long as possible.

\subsection{Engineering issues}

The engineering issues come from exposure of power plant materials to degradation phenomena and/or environmental exposure conditions never foreseen when the plant was designed, for example:

- increased dose leading to materials embrittlement, swelling and cracking susceptibility;

- increased exposure of materials and structures to operation at high temperature and pressure, leading to:

- higher than anticipated creep damage;

- material embrittlement;

- material properties degradation due to thermal effects;

- increased susceptibility to Environmental Assisted Cracking.

- Increased numbers of thermal and pressure cycles leading to increased fatigue;

- a switch from traditional base-load operations to loadfollowing operations [2] leading to increased temperature and pressure cycling.

\subsection{Legislative issues}

Irrespective of European country, operation of nuclear power plant is under-pinned by a safety case, justifying the 
safety of operation, and approved by a regulatory authority. The validity of safety cases often takes advantage of assessments to available codes and standards (e.g. ASME, ISO). The attraction is the standards' internationally agreed status, underpinned by significant collaborative discussions. Generally, the requirements of standards are stable, since they require significant international consensus to revise, but occasionally significant iterations in standards can emerge which require attention in safety submissions.

Thus, creation of challenge to long-term operation can arise:

- when an assessor needs to justify operation beyond the scope of available standards;

- when a significant update to available standards necessitates safety case revision if the case is to remain compliant with the standard.

\subsection{Safety issues}

Public perceptions of nuclear power as an environmentally clean source of electricity are improved today, compared with a few decades ago. However, awareness of the significant consequences possible following nuclear accidents is also very strong given some high profile events such as Fukoshima, Chernobyl, Three Mile Island and the Windscale fire. Therefore, high reliability assurance of safety is rightly demanded for nuclear power plant. For this reason, assurance of safety sits behind all of the issues discussed above. It also drives the need for high confidence in predictions of material degradation or structural integrity.

\section{Long-term operation challenges and the advances gained from the project portfolio}

There are a number of challenges arising from the issues described above. Some are mainly relevant to new plant, others to older operating plant, and some to both situations. Each challenge is described in the following sub-sections, together with examples of how the challenge has been met by the project portfolio covered by this paper.

\subsection{Materials performance over at least 60 years}

This challenge is, how to predict material performance over at least 60 years, when there is no experience of such long exposures? It is relevant to new build and to current plant. Four of the projects covered by this paper have tackled this challenge:

- INCEFA + focuses on improving predictability of fatigue endurance for austenitic stainless steel, in light water reactor environment, over extended operation. Tests are accelerated, compared to plant conditions, through cyclic loading that is more frequent than would occur in plant. However, care is taken to ensure that loading rates are not so fast as to render environmental effects irrelevant, since this would invalidate the results for supporting long-term operation. Statistical significance for the findings is assured through a large test matrix, adherence to common test materials and finishes, common agreed testing methods, and consistent data recording.

- SOTERIA tackles long-term radiation damage to Reactor Pressure Vessel steels (which can suffer embrittlement), and Reactor Internals (which can become susceptible to Irradiation Assisted Stress Corrosion Cracking, IASCC). There is emphasis in this project on developing mechanistic understanding of the degradation processes, and using this to develop models that can be used to extrapolate to long-term operation. The understanding in this project derives from detailed examination of materials at various scales from subatomic to whole test specimens.

- MEACTOS is tackling the sensitivity of Stress Corrosion Cracking (SCC) to surface finish. The goal is creation of practical guidelines on the creation of surface finishes able to have maximum resistance to SCC over extended operation. Whilst not specifically targeting extrapolation of susceptibility to the long-term, the programme will determine optimum surface finishes that can then be proven through accelerated testing. Optimisation of accelerated test methods is one of the objectives of this project in order to allow it to deliver its primary objective. Since surface finish is of interest to both MEACTOS and INCEFA +, there has been collaboration between these projects, particularly regarding consistent creation and measurement of surface finishes.

- Several of the pilot projects performed under NUGENIA + were focussed on materials performance. McSCAMP, MICRIN + and ASATAR separately looked at effects of machining on SCC, and at different types of SCC test and their suitability for accelerated testing; the larger MEACTOS project benefitted from these pilot projects. APLUS delivered standard protocols for analysis of atom probe data that were available to SOTERIA, which has used atom probe tomography to investigate microstructure evolution under irradiation of RPV steels. AGE60+ investigated use of common test databases, with particular focus on data collation relating to RPV embrittlement and SCC of reactor internals. Both these subject areas have been progressed further during SOTERIA, whilst INCEF A+'s focus on use of a common long-term test database is also consistent with the recommendations of AGE60+.

- A recurrent requirement for being able to justify extended materials performance is the availability of statistically significant data, able to demonstrate the trends in materials behaviour necessary for extrapolation to long lives. For INCEFA+, SOTERIA and MEACTOS, the resource requirements for the testing are significant and beyond the capabilities of any one laboratory. Furthermore, there remain significant differences in opinion as to how accelerated testing should be done. The assembly of focussed consortia, comprising the majority of European expertise, enables development of robust test strategies that can be better defended under scrutiny from outside Europe, and from regulatory bodies. The combining of resources also helps maximise the statistical significance of the project findings. It is notable that all three projects have developed interna- 
tional links beyond Europe (especially in the USA and Japan) that also help ensure best practice and provide access to additional supporting data.

- The NUGENIA + pilot projects were small (by definition), with small consortia. Nonetheless, through exposure to peer scrutiny via NUGENIA, the ideas generated for possible extended work could be properly evaluated for maximum benefit.

\subsection{Materials choice for long-term operation}

This challenge is relevant to new-build plant. The work described in the preceding section is relevant. In particular, the work being done by INCEFA + and MEACTOS will help plant designers choose surface finishes best able to mitigate either environmental fatigue or SCC. It is also notable that MEACTOS is testing both austenitic stainless steels and nickel-based alloys, and INCEFA + is testing some stabilised materials for comparison with the standard 304 stainless steel used for most of its tests.

Other than these examples, it is true that the projects mostly concentrate on limited material selections. However, development of mechanistic understanding does offer the chance of extrapolating findings to other materials, albeit with the need to do confirmatory testing eventually. SOTERIA and MEACTOS, in particular, are both significantly increasing mechanistic understanding and so their findings are relevant to this challenge.

\subsection{Design code fitness for purpose}

As described above, plant safety cases, as much as possible, take advantage of codes and standards. However, circumstances do arise, for both new and operating plant, when assessors have to consider safety justification for conditions beyond the scope of such references. Challenges are as follows:

- How to extrapolate beyond the scope of codes? For example, some codes prescribe minimum allowable thicknesses (MAT). However, for localised defects, tolerable penetration can be allowed to exceed MAT. Assessments to justify such departures must obviously be robust and defendable.

- How to alleviate excessive code conservatism that is not considered relevant? For example, many codes have evolved over significant time, with factors of safety introduced over the years for a variety of reasons, often due to emerging research. Sometimes, conservatisms can compound. Whilst conservatism is retained with this approach, it can be excessively pessimistic for some circumstances. For an assessor to justify departure from accepted advice, there is (rightly) a strong requirement for reliable, statistically significant evidence. follows:

The project portfolio has tackled these challenges as

- INCEFA + was set up in direct response to emergent United Stated Regulatory Commission (USNRC) guidance to assume an environmental penalty for assessments of endurance in light water reactor (LWR) conditions. This penalty applies to design curves for fatigue endurance in air, which already contain allowances for effects such as surface condition. There is evidence to show that some effects already allowed for in air design curves do not have the same effect in LWR conditions; however, the quantity and statistical significance of available data was insufficient to justify departure from USNRC recommendations. INCEFA + tackles three sensitivities, surface finish, hold time and mean stress, and determines how these vary between air and LWR environments. By combining 13 European laboratory resources, the project is creating the quantity of data needed for a robust response on these issues. Furthermore, by agreement of common test protocols, data formats, and use of common materials and specimen conditions, the project reduces scatter leading to further statistical reliability.

- Building on the NUGENIA+ pilot projects, MEACTOS tackles established practice to control surface finish of components in terms of only surface roughness. The belief is that newly available machining techniques offer the potential for SCC susceptibility mitigation. The project will produce guidelines for designers to use to specify surface finish requirements. The validity of accelerated SCC testing methods can be questioned, and furthermore resource requirements for SCC testing can be large. Bringing together leading European expertise helps (a) ensure best practice, and (b) deliver statistical significance. Inclusion of industrial machining expertise also maximises the likely relevance and usefulness of the project guidelines.

- ATLAS + is developing improved methods for prediction of ductile tearing for large defects in components, and for undertaking leak-before-break (LBB) assessments of piping components. The project will quantify the uncertainties and confidence in these methods using probabilistic approaches. Such assessments are specialised and beyond the scope of basic design codes; thus, high confidence is a requirement for use of such techniques. The ATLAS + strategy is an assessment programme examining residual stress effects, validated using a comprehensive multiscale testing programme. The test programme is demanding of resources, since it includes large scale testing as well as conventional lab specimen tests. Furthermore, the assessment methodologies are specialised. Thus, a major ATLAS+ advantage is the assembled consortium. This provides the test resources necessary, and also combines leading European experts for this subject. The result promises to be highly significant and likely to be positively received internationally.

- The NUGENIA + pilot project DEFI-PROSAFE explored potential benefits of a probabilistic integrity assessment approach for Reactor Pressure Vessel assessment. Results suggested possible significant positive impact potential for margin to long-term operation. These findings are available for building on at some stage.

\subsection{Justification for operation of structures}

This applies to operational and new-build plant. Obviously, materials understanding, combined with code familiarity are both important to meet this challenge. However, 
structural response must also be tackled, in particular there must be confidence in the possible failure mode. Assessors must demonstrate that failure would be benign rather than catastrophic (e.g LBB).

ATLAS + and the earlier NUGENIA + pilot project DEFI-PROSAFE are both clearly focussed on this challenge, one for pipes, and one for RPV's.

\subsection{Threat mitigation through inspection}

This applies to all stages of plant life. Once degradation is credible, the next challenges are how quickly cracks may propagate, and how reliably propagation could be detected prior to it becoming problematic? Each of the four full projects, plus several NUGENIA + pilot projects, deliver useful advances in understanding of degradation timescales.

For flaw detection, the NUGENIA + pilot projects REDUCE and MAPAID are relevant. MAPAID considered the reliability of Phased Array ultrasonic inspection of dissimilar metal welds. REDUCE evaluated the reduced risk possible through use of in-service inspection. These projects were pre-cursors to the projects $\mathrm{NOMAD}^{6}$ and ADVISE. ${ }^{7}$ These projects are not within the scope of this paper.

\subsection{Expertise availability}

Many European organisations have skewed staff demographics resulting from limited recruitment during the 1990s in particular. The result is a pool of expertise at, or already beyond, retirement age, with limited expertise in the successor staff. Development of the next generation of experts is important to maintain capability to meet the challenges to long-term operation. Expertise availability challenge also arises from reduced interest of the new generations in nuclear energy. Some analysts suggest the cause is competition from renewable energy sources. However, although nuclear accidents have created negative reaction, growing energy demand and non-generation of greenhouse gases also keeps nuclear energy as a "green" option, which should help public perception. Perhaps, the problem comes from nuclear sector conservatism, from which overprotection has slowed technological innovation.

The most attractive professional careers are those with highest technological content. Many technologies and innovative approaches for fabrication, repair and joining are currently available in non-nuclear industries, but are not addressed in nuclear codes and standards or endorsed by regulatory bodies. This difficulty about the adoption of technologies threatens the nuclear industry with technological obsolescence. Restoring the nuclear industry's lead in technology development is important to recover attractiveness for working in this sector.

\footnotetext{
${ }^{6}$ This project has received funding from the Euratom H2020 programme under grant agreement No 755330.

${ }^{7}$ This project has received funding from the Euratom H2020 programme under grant agreement No 755500.
}

Fortunately, dissemination and sponsoring of students is encouraged in EC supported projects. Furthermore, the projects in this paper will significantly advance understanding in some technologically advanced subjects. Examples of this are as follows:

INCEFA+

- A public website is maintained, along with a ResearchGate presence and a Twitter account. Significant traffic demonstrates interest in INCEFA+.

- The project is presented at international conferences (e.g., ASME Code Week 2017, NPFA 2017, ASME PVP2017 and 2018, PLiM2017, annual NUGENIA Forums, Fracture Fatigue and Wear 2018, 22nd European Conference on Fracture). Project presentations are committed for 2019 and 2020.

- Project special sessions have taken place at the XVIII International Colloquium on Mechanical Fatigue of Metals (ICMFM XVIII, September 2016, Gijón, Spain), and at the ASME PVP2018 conference in July 2018 in Prague, Czech Republic.

- The dissemination activity has led to nine international scientific papers indexed in Scopus; the events expected for 2019 and 2020 will increase this number. Also, a third project session is agreed to take place at ASME PVP2020.

- The first Seminar and Workshop Dissemination event was in June 2018 in Santander, Spain. This provided an introduction to fatigue and environmental fatigue phenomena, and to the treatment of them for different industries, through presentations by experts from industrial and research organisations. The seminar was designed for $\mathrm{PhD}$ and Masters students, professional engineers and researchers new to the field, or experienced researchers and engineers wishing to update their knowledge and share experiences. The event was attended by about 70 people and feedback was excellent. - A second dissemination workshop, designed to appeal to established researchers, is planned for June 2020 in Aixen-Provence, France.

\section{SOTERIA}

- The demographic challenge in SOTERIA is mainly addressed through the dissemination activities (training school and workshops).

- The SOTERIA Training School was held in September 2018 in Valencia (Spain), with the aim of transferring and preserving the knowledge about nuclear reactor pressure vessel and internal materials degradation mechanisms to students, post-docs and early career professionals, as well as to scientists and engineers working on these areas. The school hosted 60 participants, including students, lecturers and organisers, with a share of $20 \%$ women and $80 \%$ men. While most students were in their early career, many "advanced" students also attended. The participants came from 29 different organisations, distributed in 13 different countries. About $80 \%$ of the organisations represented at the school were European, but there was also presence from Argentina, Rep. of Armenia, Mexico, Ukraine and Switzerland. Most participants came from research and development $(\mathrm{R} \& \mathrm{D})$ organisations although utilities, safety authorities 
and technical safety organisations were also represented. The programme, focused on the effects of irradiation on RPV and internals materials, with emphasis on a longterm operation approach, comprised three days of lectures and two days of interactive sessions, with hands-on demonstrations, working with the new version of the SOTERIA platform. From analysis of the questionnaire filled in by school attendees, it is clear that the training school was positively appreciated.

- The SOTERIA Mid-term Workshop was in April 2018 in Prague. The workshop was a great opportunity for dissemination of important results achieved in SOTERIA. It was also useful to facilitate interchange of ideas and experiences with the full Nuclear Research Community, especially with NUGENIA members. On the last day, a Joint Technical Session, with other related NUGENIA projects (NOMAD, ADVISE, INCEFA+, ATLAS+ and MEACTOS), was held to exchange information and available results.

- The SOTERIA Final Workshop is in June 2019 in Miraflores de la Sierra (Madrid). The objective is to disseminate project final results among nuclear research and industrial communities, and particularly end-users, as well as identifying future research needs. The workshop will be a forum for regulators, user groups, experts and industry, to exchange information and experiences on radiation effects on nuclear power plant components.

\section{MEACTOS}

- An objective of MEACTOS is to reduce technological obsolescence associated with the nuclear industry, evaluating the applicability of procedures for machining/surface modification of materials that have shown their effectiveness in other industrial sectors.

- MEACTOS is committed to dissemination and exploitation of results, and has created the role of exploitation manager to further this. This manag er has responsibility for finding the best ways to exploit project results, for coordinating exploitation-related issues within the Consortium, such as patents, licenses, diffusions activities, and for coordinating possible negotiations concerning exploitation issues between the Consortium and external partners.

- Actions to introduce nuclear technology to a new generation of professional are:

- presentation of project contents in different nuclear forums of participating countries;

- co-organize a summer school in cooperation with European corrosion federation NuCoss, to be in Slovenia in 2019 with expected attendance of 40 participants;

- create a web page to inform about the project, activities and events;

- formation of at least two new PhDs;

- maximize the interest and impact in the stakeholders, creating an End User Group (EUG), to which three new organizations have joined.

\section{ATLAS+}

- The knowledge transfer seminar with the title "Seminar on Piping Issues in ATLAS + (SEPIA)" was organized in October 2018 in Ljubljana. 37 people attended. The aim was to introduce and educate colleagues, new in the field, in the ATLAS + technical topics. The discussions, and questions and answers, after the presentations demonstrated great interest. Feedback from attendees after the seminar was positive and they expressed the wish to repeat this type of activity. Abstracts and presentations were provided to all participants.

- ATLAS + members disseminated first results at the ASME PVP2018 conference, in July 2018 in Prague. One session with four presentations was organised under the topic of European programs in structural integrity. Papers are planned for PVP2019. PVP papers appear in conference proceedings.

- The ATLAS + disseminations will be able to be followed on a website.

- ATLAS+ has nine training missions, where new researchers can visit another organisation. The goal is learning and sharing knowledge in ATLAS+ topics.

- A training book on the lessons learnt in ATLAS+ and summary of the final seminar is published at the end of the project.

\section{NUGENIA +}

- NUGENIA + pilot projects were small, and so major dissemination activities within each project were limited. However, through NUGENIA, there has been significant dissemination of NUGENIA + results. The pilot projects were presented and discussed at a final workshop in 2016 in Helsinki, Finland. This was open to all NUGENIA members. Ever since, it is still possible to learn about NUGENIA + projects through the NUGENIA website; where the details provide contacts if more details are sought.

- A major objective for NUGENIA is the building of knowledge and expertise in Europe. Recent examples of success in this include (a) provision of grants to facilitate short secondments of young researchers to other organisations, and (b) organising a paper competition for PhD students at the NUGENIA 2019 Forum, through which the students gained exposure to industry experts.

\section{Remaining challenges}

The NUGENIA + pilot projects were small, and intended to demonstrate the benefits possible through more work. Thus, remaining challenge from these projects was inevitable and varied.

For the four full projects, the likely remaining challenge varies as follows:

- By its end, INCEFA + will have delivered advances in understanding of the sensitivities of fatigue endurance to surface finish, hold time and mean stress in both air and LWR environments. This will be mainly for a single heat of 304 stainless steel; thus, understanding of the effects of 
material variability will remain a challenge, albeit not a serious one given low variability evident in literature for austenitic stainless steels. Regarding test condition sensitivities, the project has focussed on four, and so others will remain. Of these, the dominant remaining challenge will be how laboratory findings translate to full component scale; in fact, plans are developing for the consortium to possibly continue by addressing this knowledge gap next.

- SOTERIA's multi-scale approach to develop understanding of irradiation effects on degradation of RPV and Internals materials will deliver advances mechanistically. However, largely due to the high cost of the tests being done, the actual number of data points generated will be limited. Hence, statistical significance will remain a challenge. Furthermore, data accessibility for the long-term from this and predecessor projects is a challenge affecting usefulness of project findings for plant assessors. Building on INCEFA + experiences, the SOTERIA consortium proposes to focus on this challenge after the project ends. Meanwhile, accumulation of IASCC test evidence and understanding is also proposed to continue in a parallel possible project.

- The main MEACTOS focus is determination of optimum machining methods for SCC mitigation. As noted, the collection of a powerful consortium comprising experts in understanding and testing, and machining, promises impressive advances. However, once an optimum machining method is determined, it is likely that focussed testing to parameterise sensitivity to SCC for that method, for a variety of candidate materials, will be needed. This will support statistical substantiation sufficient for the guidance to become definitive.

- The position for ATLAS+ is different to the other projects, since the objective for the project is to deliver assessment methodologies validated using a multiscale test programme. At this stage it is not so straightforward to define the remaining challenges. Clearly, data availability into the future must be a concern, as it is for SOTERIA. Also, there will remain knowledge gaps to be pursued.
The on going nature of these research streams could perhaps indicate problems realising the project benefits. However, the projects' influence on international research and development has been demonstrated through interest in engaging with the projects from the USA and Far East in particular. Two examples of this are (a) data sharing agreements being set up by INCEFA + with USNRC, EPRI and JNRA, and (b) user groups set up for SOTERIA and MEACTOS, showing active industrial engagement and interest.

\section{Conclusions}

Safety assurance through advances in long-term operation requires research and development activities that tackle, extended period materials performance, selection of materials for new plant, improvements to design and assessment codes, structural performance, mitigation of risk through inspection, and expertise availability. Since activities to gain advances in these areas are demanding in terms of resource needed, either because of the cost of testing, or because of the volume of data required for statistical significance, it follows that the best advances are when expert organisations combine forces. The EC support for research and development activities provides funding to enable coordinated activities to be performed by expert consortia. The advantages this enables are demonstrated by reference to developments arising from the FP7 NUGENIA + pilot projects, and from the running Horizon2020, INCEFA+, SOTERIA, MEACTOS and ATLAS + projects. This paper also postulates the challenges likely to remain when these projects have ended.

\section{References}

1. World Nuclear Association, Nuclear Power in the European Union, July 2018, http://www.world-nuclear.org/informa tion-library/country-profiles/others/european-union.aspx

2. A. Lokhov, OECD. Load-following with nuclear power plants. NEA updates, NEW News 2011-No. 29.2, https://www.oecdnea.org/nea-news/2011/29-2/nea-news-29-2-load-followinge.pdf

Cite this article as: Kevin James Mottershead, Christian Robertson, Sebastian Lindqvist, Francisco Javier Perosanz Lopez, Eija Karita Puska, Safety assurance through advances in long-term operation, EPJ Nuclear Sci. Technol. 6, 44 (2020) 